NISSUNA UMANA INVESTIGAZIONE SI PUO DIMANDARE VERA SCIENZIA S'ESSA NON PASSA PER LE MATEMATICHE DIMOSTRAZIONI LEONARDO DA VINCI

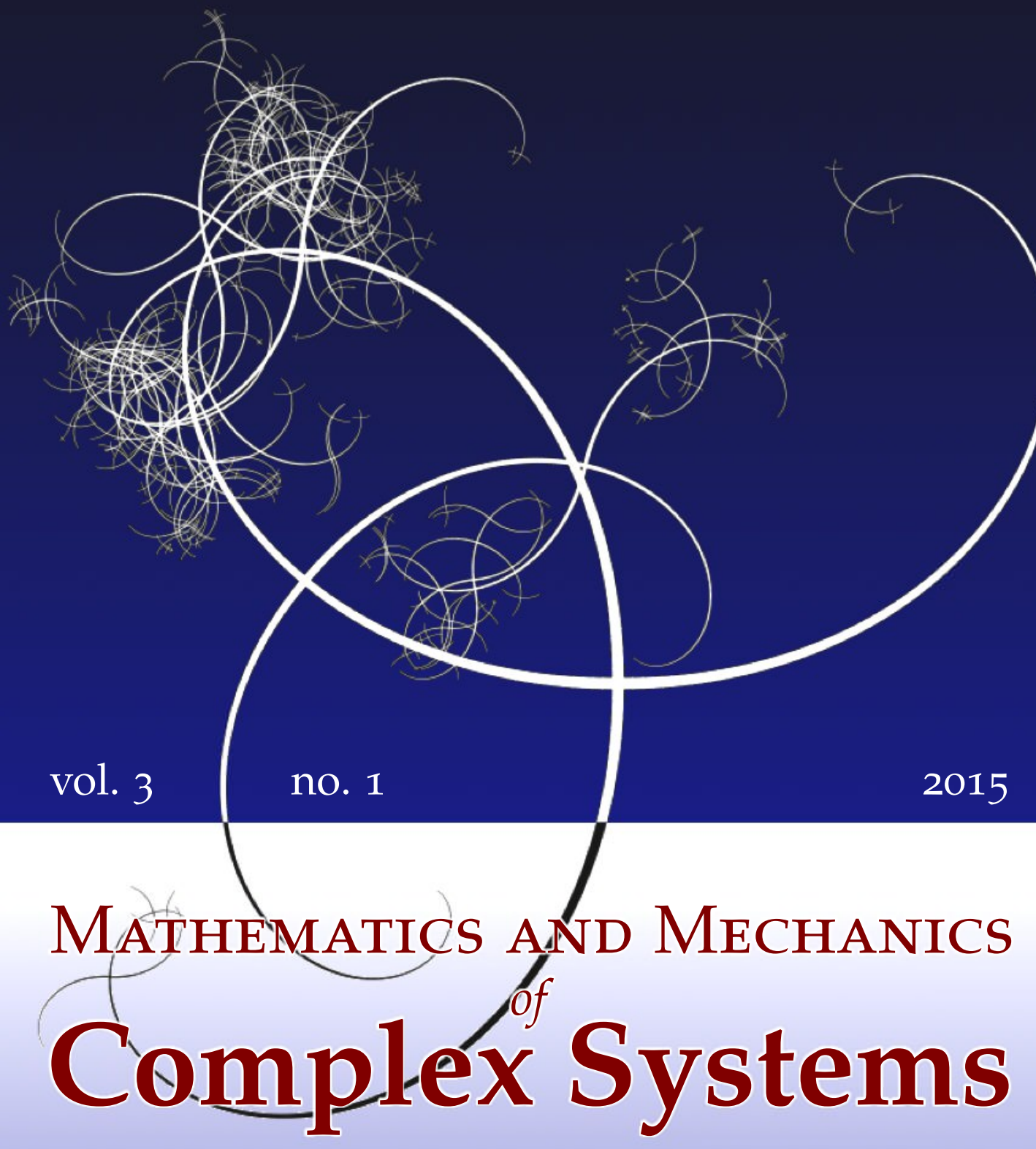

MiroslaV ŠILHAVÝ

ON THE APPROXIMATION THEOREM

FOR STRUCTURED DEFORMATIONS FROM $B V(\Omega)$ 


\title{
ON THE APPROXIMATION THEOREM FOR STRUCTURED DEFORMATIONS FROM $B V(\Omega)$
}

\author{
MiroslaV ŠILHAVÝ
}

\begin{abstract}
This note deals with structured deformations introduced by Del Piero and Owen. As treated in the present paper, a structured deformation is a pair $(\boldsymbol{g}, \boldsymbol{G})$ where $\boldsymbol{g}$ is a macroscopic deformation giving the position of points of the body and $\boldsymbol{G}$ represents deformations without disarrangements. Here $\boldsymbol{g}$ is a map of bounded variation on the reference region $\Omega$, and $\boldsymbol{G}$ is a Lebesgue-integrable tensorvalued map. For structured deformations of this level of generality, an approximating sequence $\boldsymbol{g}_{k}$ of simple deformations is constructed from the space of maps of special bounded variation on $\Omega$, which converges in the $L^{1}(\Omega)$ sense to $(\boldsymbol{g}, \boldsymbol{G})$ and for which the sequence of total variations of $\boldsymbol{g}_{k}$ is bounded. The condition is optimal. Further, in the second part of this note, the limit relation of Del Piero and Owen is established on the above level of generality. This relation allows one to reconstruct the disarrangement tensor $\boldsymbol{M}$ of the structured deformation $(\boldsymbol{g}, \boldsymbol{G})$ from the information on the approximating sequence.
\end{abstract}

\section{Introduction and results}

This paper deals with the geometry of deformation of nonclassical continua modeled as media capable of (first-order) structured deformations introduced by Del Piero and Owen $[1993 ; 1995] .{ }^{1}$ The main objective of the theory of structured deformations is to describe how a continuous body with microstructure will deform under the applied forces.

In the original setting [Del Piero and Owen 1993; 1995], a structured deformation is a triplet $(\mathcal{K}, \boldsymbol{g}, \boldsymbol{G})$ of objects whose nature will now be roughly described. The set $\mathcal{K}$, the crack site, is a subset of vanishing Lebesgue measure of the reference region $\Omega$, the map $g: \Omega \sim \mathcal{K} \rightarrow \mathrm{R}^{3}$, the deformation map, is piecewise continuously differentiable and injective, and $\boldsymbol{G}$ is a piecewise continuous map

\section{Communicated by Gianpietro Del Piero.}

MSC2010: primary 74R99; secondary 74A05.

PACS2010: 81.40.Lm.

Keywords: structured deformation, fracture, approximations, maps of bounded variation, maps of special bounded variation.

${ }^{1}$ The reader is referred to the proceedings [Del Piero and Owen 2004] and to the recent survey [Baía et al. 2011] for additional references and for further developments. 
from $\Omega \sim \mathcal{K}$ to the set of invertible second-order tensors describing deformation without disarrangements. The following "accommodation inequality" is assumed:

$$
0<m \leq \operatorname{det} \boldsymbol{G} \leq \operatorname{det} \mathrm{D}_{\mathrm{a}} \boldsymbol{g}
$$

in $\Omega \sim \mathcal{K}$, with $m$ a suitable constant, where $\mathrm{D}_{\mathrm{a}} \boldsymbol{g}$ is the classical derivative of $\boldsymbol{g}$ where it exists. ${ }^{2}$ Within this context, a classical deformation is the triplet $(\mathcal{K}, \boldsymbol{g}, \mathrm{D} \boldsymbol{g})$ with $\boldsymbol{g}$ a continuously differentiable injective deformation function and with $\boldsymbol{G}:=$ $\mathrm{D} \boldsymbol{g}=\mathrm{D}_{\mathrm{a}} \boldsymbol{g}$ the deformation gradient, where $\mathrm{D}$ denotes the derivative (gradient) operation on differentiable maps. A more general class of structured deformations is provided by simple deformations, which are triples $\left(\mathcal{K}, \boldsymbol{g}, \mathrm{D}_{\mathrm{a}} \boldsymbol{g}\right)$ where $\boldsymbol{g}$ is only piecewise-smooth injective with jump discontinuities describing partial or full separation of pieces of the body and $\boldsymbol{G}:=\mathrm{D}_{\mathrm{a}} \boldsymbol{g}$. In view of these classes, where $\boldsymbol{G}$ coincides with the deformation gradient, in the general case, the tensor of deficit

$$
\boldsymbol{M}=\mathrm{D}_{\mathrm{a}} \boldsymbol{g}-\boldsymbol{G}
$$

measures the departure of $(\mathcal{K}, \boldsymbol{g}, \boldsymbol{G})$ from the simple deformation $\left(\mathcal{K}, \boldsymbol{g}, \mathrm{D}_{\mathrm{a}} \boldsymbol{g}\right)$.

A substantial step towards a concrete interpretation of the tensor $\boldsymbol{G}$ is offered by the approximation theorem [Del Piero and Owen 1993, Theorem 5.8]. That theorem shows that each structured deformation $(\mathcal{K}, \boldsymbol{g}, \boldsymbol{G})$ is a limit of a suitable sequence of simple deformations $\left(\mathcal{K}_{k}, \boldsymbol{g}_{k}, \mathrm{D}_{\mathrm{a}} \boldsymbol{g}_{k}\right)$ in the sense that

$$
\mathcal{K}_{k} \rightarrow \mathcal{K}, \quad \boldsymbol{g}_{k} \rightarrow \boldsymbol{g}, \quad \text { and } \quad \mathrm{D}_{\mathrm{a}} \boldsymbol{g}_{k} \rightarrow \boldsymbol{G}
$$

with suitably defined convergences of the objects in (2). I note that the nontrivial feature of the proof of the approximation theorem lies in proving the injectivity of $\boldsymbol{g}_{k}$. Moreover, Del Piero and Owen [1993] prove the following the limit relation for the tensor $\boldsymbol{M}$ :

$$
\boldsymbol{M}(\boldsymbol{x})=\lim _{\rho \rightarrow 0} \lim _{k \rightarrow \infty}(4 \pi / 3)^{-1} \rho^{-3} \int_{J\left(\boldsymbol{g}_{k}\right) \cap \mathbf{B}(\boldsymbol{x}, \rho)}\left[\boldsymbol{g}_{k}\right] \otimes \boldsymbol{n}_{k} d \mathcal{H}^{2},
$$

valid for any sequence (not just the one constructed in the proof of the approximation theorem) $\left(\mathcal{K}_{k}, \boldsymbol{g}_{k}, \mathrm{D}_{\mathrm{a}} \boldsymbol{g}_{k}\right)$ satisfying (2) and any $\boldsymbol{x} \in \Omega \sim \mathcal{K}$, where $\mathbf{B}(\boldsymbol{x}, \rho)$ is the open ball of center $\boldsymbol{x}$ and radius $\rho, J\left(\boldsymbol{g}_{k}\right)$ is the set of all points of (jump) discontinuity of $\boldsymbol{g}_{k},\left[\boldsymbol{g}_{k}\right]$ is the jump of $\boldsymbol{g}_{k}$ at the points of $\boldsymbol{J}\left(\boldsymbol{g}_{k}\right), \boldsymbol{n}_{k}$ is the normal to $J\left(\boldsymbol{g}_{k}\right)$, and $\mathcal{H}^{2}$ is the area measure.

To apply the relaxation techniques of the calculus of variations, Choksi and Fonseca [1997] later enlarged the space of structured deformations to contain all

${ }^{2}$ Later we shall identify $\mathrm{D}_{\mathrm{a}} \boldsymbol{g}$ with the absolutely continuous part of the derivative of a map $\boldsymbol{g}$ of bounded variation. 
pairs $(\boldsymbol{g}, \boldsymbol{G})$ where $\boldsymbol{g}$ is in ${ }^{3} \operatorname{SBV}\left(\Omega, \mathrm{R}^{m}\right)$ and $\boldsymbol{G}$ is in $L^{1}\left(\Omega, \mathrm{M}^{m \times n}\right)$. Here $m$ and $n$ are positive integers, the dimensions of the spaces $\mathrm{R}^{m}$ and $\mathrm{R}^{n}$ of dependent and independent variables, respectively. Thus, in addition to weaker regularity, the authors relax the injectivity requirement and put the crack site $\mathcal{K}$ equal to $\varnothing$. (The cracks are described by the omnipresent discontinuities of $\boldsymbol{g}$.)

Choksi and Fonseca [1997, Theorem 2.12] prove the following version of the approximation theorem, which is stated here in a slightly rephrased form as explained below:

Theorem 1.1. Let $\Omega \subset \mathrm{R}^{n}$ be a bounded open set, and let $(\boldsymbol{g}, \boldsymbol{G}) \in L^{1}\left(\Omega, \mathrm{R}^{m}\right) \times$ $L^{1}\left(\Omega, \mathrm{M}^{m \times n}\right)$. Then there exists a sequence $\boldsymbol{g}_{k}$ in $\operatorname{SBV}\left(\Omega, \mathrm{R}^{m}\right)$ such that

$$
\boldsymbol{g}_{k} \rightarrow \boldsymbol{g} \text { in } L^{1}\left(\Omega, \mathrm{R}^{m}\right) \quad \text { and } \mathrm{D}_{\mathrm{a}} \boldsymbol{g}_{k}=\boldsymbol{G} \text { over } \Omega .
$$

Here $\mathrm{D}_{\mathrm{a}} \boldsymbol{g}_{k}$ is the absolutely continuous part of the generalized derivative of $\boldsymbol{g}_{k}$. The statement of [Choksi and Fonseca 1997, Theorem 2.12] is narrower since (a) the authors assume, in accord with the overall framework of their paper, that $\boldsymbol{g}$ is in $\operatorname{SBV}\left(\Omega, \mathrm{R}^{m}\right)$ and (b) since they replace the equality (4) $)_{2}$ by the weak* convergence in the sense of measures (although they say that they will prove the equality). Their proof also shows that $g \in L^{1}\left(\Omega, \mathrm{R}^{m}\right)$ suffices.

In connection with this generality, the question arises: what additional information beyond (4) can be imposed on the sequence $\boldsymbol{g}_{k}$ if it is known that $\boldsymbol{g}$ belongs to the smaller space $B V\left(\Omega, \mathrm{R}^{m}\right)$ or even to $S B V\left(\Omega, \mathrm{R}^{m}\right)$ ? An answer, one of the two goals of this note, given in the subsequent theorem, is proved for reference regions represented by admissible domains (which is a mild restriction on $\Omega$, satisfied, e.g., by all open sets with lipschitzian boundary). ${ }^{4}$

Theorem 1.2 (approximation theorem). If $\Omega$ is an admissible domain in $\mathrm{R}^{n}$ and $(\boldsymbol{g}, \boldsymbol{G}) \in B V\left(\Omega, \mathrm{R}^{m}\right) \times L^{1}\left(\Omega, \mathrm{M}^{m \times n}\right)$, then there exists a sequence $\boldsymbol{g}_{k} \in \operatorname{SBV}\left(\Omega, \mathrm{R}^{m}\right)$ such that in addition to (4) the total variation $\mathrm{M}\left(\mathrm{D} \boldsymbol{g}_{k}\right)$ of $\boldsymbol{g}_{k}$ satisfies

$$
\sup \left\{\mathrm{M}\left(\mathrm{D} \boldsymbol{g}_{k}\right): k=1, \ldots\right\}<\infty
$$

hence, we have the following convergence (without passing to a subsequence):

$$
\mathrm{D} \boldsymbol{g}_{k} \rightarrow^{*} \mathrm{D} \boldsymbol{g} \text { in } \mathcal{M}\left(\Omega, \mathrm{M}^{m \times n}\right) \text {. }
$$

${ }^{3} \mathrm{I}$ use the standard notations for function spaces throughout this introduction: thus, $B V\left(\Omega, \mathrm{R}^{n}\right)$ and $\operatorname{SBV}\left(\Omega, \mathrm{R}^{n}\right)$ are spaces of $\mathrm{R}^{m}$-valued maps on $\Omega$ of bounded variation and of special bounded variation, and $L^{1}\left(\Omega, \mathrm{R}^{m}\right)$ and $L^{1}\left(\Omega, \mathrm{M}^{m \times n}\right)$ are spaces of (Lebesgue-)integrable $\mathrm{R}^{m}$ - or $\mathrm{M}^{m \times n}$ valued maps on $\Omega$. $\mathcal{M}\left(\Omega, \mathrm{M}^{m \times n}\right)$ is the space of $\mathrm{M}^{m \times n}$-valued measures on $\Omega$. The reader is referred to Sections 2 and 3 below for detailed definitions.

${ }^{4}$ See Lemma 5.1 on page 93. 
Thus, the extra information stemming from the inclusion $g \in B V\left(\Omega, \mathrm{R}^{m}\right)$ is (5). It is easy to see that, conversely if $(\boldsymbol{g}, \boldsymbol{G}) \in L^{1}\left(\Omega, \mathrm{R}^{m}\right) \times L^{1}\left(\Omega, \mathrm{M}^{m \times n}\right)$ is a pair satisfying (4) and (5) with $\boldsymbol{g}_{k} \in S B V\left(\Omega, \mathrm{R}^{m}\right)$, then necessarily $\boldsymbol{g} \in B V\left(\Omega, \mathrm{R}^{m}\right)$; in this sense, (5) is optimal. (Both directions are very intuitive.) The proof of the boundedness in the approximation theorem is based on the observation in Lemma 5.1 below, but otherwise the construction of the sequence essentially follows that of Choksi and Fonseca. ${ }^{5}$

The second goal of the present note is to give an analog to the limit relation (3) in the setting of maps of bounded variation.

Theorem 1.3 (the limit relation). Let $\Omega$ be a bounded open subset of $\mathrm{R}^{n}$, let $(\boldsymbol{g}, \boldsymbol{G}) \in B V\left(\Omega, \mathrm{R}^{m}\right) \times L^{1}\left(\Omega, \mathrm{M}^{m \times n}\right)$, and let $\boldsymbol{g}_{k} \in S B V\left(\Omega, \mathrm{R}^{m}\right)$ be a sequence satisfying

$$
\boldsymbol{g}_{k} \rightarrow \boldsymbol{g} \text { in } L^{1}\left(\Omega, \mathrm{R}^{m}\right), \quad \mathrm{D}_{\mathrm{a}} \boldsymbol{g}_{k} \rightarrow \boldsymbol{G} \text { in } L^{1}\left(\Omega, \mathrm{M}^{m \times n}\right),
$$

and (6) (in particular, let $\boldsymbol{g}_{k}$ be the sequence from Theorem 1.2). Then there exists a subsequence of $\boldsymbol{g}_{k}$ (not relabeled) such that the tensor $\boldsymbol{M}$ (see (1)) satisfies



for almost every point $\boldsymbol{x}$ of $\Omega$.

Here ess $\lim _{\rho \rightarrow 0}$ is the essential limit as $\rho \rightarrow 0$, i.e., the limit neglecting an exceptional set of $\rho$ 's of vanishing Lebesgue measure. ${ }^{6}$ Further, $\kappa_{n}$ is the volume of the unit ball in $\mathrm{R}^{n}, J\left(\boldsymbol{g}_{k}\right)$ is the set of all points of jump discontinuity of $\boldsymbol{g}_{k}$, $\left[\boldsymbol{g}_{k}\right]$ is the jump of $\boldsymbol{g}_{k}$ at the points of $J\left(\boldsymbol{g}_{k}\right), \boldsymbol{n}_{k}$ is the normal to $J\left(\boldsymbol{g}_{k}\right)$, and $\mathcal{H}^{n-1}$ is the $(n-1)$-dimensional Hausdorff measure. ${ }^{7}$

The Appendix to the present paper also outlines a proof of a weaker version of the approximation theorem that does not use Alberti's theorem mentioned above. In that version, the equality $(4)_{2}$ is replaced by the convergence $(7)_{2}$.

\section{Preliminaries, notation, and measures}

Throughout, $n$ is a positive integer, the dimension of the underlying space $\mathrm{R}^{n}$, and $m$ is a positive integer, the dimension of the target space $\mathrm{R}^{m}$. We denote by $\boldsymbol{a} \cdot \boldsymbol{b}$ the scalar product in both these spaces and by $|\cdot|$ the euclidean norm. Further, $\mathbf{M}^{m \times n}$ is the set of all linear transformations from $\mathrm{R}^{n}$ to $\mathrm{R}^{m}$. The value of $\boldsymbol{A} \in \mathrm{M}^{m \times n}$ on $\boldsymbol{x} \in \mathrm{R}^{n}$ is denoted by $\boldsymbol{A x}$. We denote by $\boldsymbol{A} \cdot \boldsymbol{B}:=\operatorname{tr}\left(\boldsymbol{A} \boldsymbol{B}^{\mathrm{T}}\right)$ the scalar product

5 In particular, Alberti's theorem [1991] (Theorem 3.7 below) is used in the same way as in [Choksi and Fonseca 1997].

${ }^{6}$ See the definition in Section 2 below.

${ }^{7}$ See Section 3 for precise definitions of these notions. 
in $\mathrm{M}^{m \times n}$, where $\boldsymbol{A}^{\mathrm{T}} \in \mathrm{M}^{n \times m}$ is the transpose of $\boldsymbol{A}$ and tr denotes the trace. We further denote by $|\boldsymbol{A}|=\sqrt{\boldsymbol{A} \cdot \boldsymbol{A}}$ the associated euclidean norm.

If $f$ is a map with domain any set $M$ and if $N \subset M$, then $f \mid N$ denotes the restriction of $f$ to $N$.

The interior, closure, and boundary of a set $M \subset \mathrm{R}^{n}$ is denoted by int $M, \operatorname{cl} M$, and bdry $M$. As in the introduction, $\mathbf{B}(\boldsymbol{x}, \rho)$ denotes the open ball in $\mathrm{R}^{n}$ of center $\boldsymbol{x}$ and radius $\rho$. The symbol $\kappa_{n}$ denotes the volume of $\mathbf{B}(\mathbf{0}, 1)$.

Throughout, let $\Omega$ be an open subset of $\mathrm{R}^{n}$, later to be restricted by additional requirements. Let $Z$ be a finite-dimensional inner-product space.

We denote by $\mathcal{L}^{n}$ the Lebesgue measure in $\mathrm{R}^{n}$ [Federer 1969, §2.6.5], and if $k$ is an integer, $0 \leq k \leq n$, we denote by $\mathcal{H}^{k}$ the $k$-dimensional Hausdorff measure in $\mathrm{R}^{n}$ [ibid., §§2.10.2-2.10.60]; recall that $\mathcal{H}^{n}=\mathcal{L}^{n}$. If $A \subset \mathrm{R}^{n}$ is a Borel set, we denote by $\mathcal{H}^{k}\left\llcorner A\right.$ the restriction of $\mathcal{H}^{k}$ to $A$, which is the measure defined by

$$
\left(\mathcal{H}^{k}\llcorner A)(B)=\mathcal{H}^{k}(A \cap B)\right.
$$

for each Borel set $B \subset \mathrm{R}^{n}$. If $A \subset \mathrm{R}^{n}$ is a Borel set and $f$ a $Z$-valued Borel map defined $\mathcal{H}^{k}$ almost everywhere on $A$, integrable with respect to $\mathcal{H}^{k}$ on $A$, then $\boldsymbol{f} \mathcal{H}^{k}\left\llcorner A\right.$ denotes the $Z$-valued measure on $\mathrm{R}^{n}$ defined by

$$
\left(\boldsymbol{f} \mathcal{H}^{k}\llcorner A)(B)=\int_{A \cap B} \boldsymbol{f} d \mathcal{H}^{k}\right.
$$

for each Borel set $B \subset \mathrm{R}^{n}$. The definitions (9) and (10) also apply to $k=n$, i.e., to $\mathcal{L}^{n} \equiv \mathcal{H}^{n}$, resulting in $\mathcal{L}^{n}\left\llcorner A\right.$ and $\boldsymbol{f} \mathcal{L}^{n}\llcorner A$.

We denote by $L^{1}(\Omega, Z)$ the set of all (classes of equivalence of) Lebesgueintegrable maps on $\Omega$ with values in $Z$; we write $|\cdot|_{L^{1}(\Omega, Z)}$ for the norm on $L^{1}(\Omega, Z)$, defined by

$$
|\boldsymbol{f}|_{L^{1}(\Omega, Z)}=\int_{\Omega}|\boldsymbol{f}| d \mathcal{L}^{n}
$$

for each $f \in L^{1}(\Omega, Z)$. We denote by $C_{0}^{\infty}(\Omega, Z)$ the set of all of indefinitely differentiable $Z$-valued maps $f$ on $\mathrm{R}^{n}$ with compact support contained in $\Omega$.

We denote by $\mathcal{M}(\Omega, Z)$ the set of all (finite) $Z$-valued measures on $\Omega$. If $\boldsymbol{\mu} \in$ $\mathcal{M}(\Omega, Z)$, we denote by $|\boldsymbol{\mu}|$ the total variation (measure) of $\boldsymbol{\mu}$, i.e., the smallest nonnegative measure on $\Omega$ such that $|\boldsymbol{\mu}(B)| \leq|\boldsymbol{\mu}|(B)$ for each Borel subset $B$ of $\Omega$. We denote by $\mathrm{M}(\boldsymbol{\mu})$ the mass of $\boldsymbol{\mu}$, defined by $\mathrm{M}(\boldsymbol{\mu})=|\boldsymbol{\mu}|(\Omega)$. A standard result is that

$$
\mathrm{M}(\boldsymbol{\mu})=\sup \left\{\int_{\Omega} \boldsymbol{f} \cdot d \boldsymbol{\mu}: \boldsymbol{f} \in C_{0}^{\infty}(\Omega, Z),|\boldsymbol{f}| \leq 1 \text { on } \Omega\right\} .
$$


We say that a measure $\mu \in \mathcal{M}(\Omega, Z)$ is supported by a Borel set $A \subset \Omega$ if $\mu(B)=0$ for every Borel set $B \subset \Omega$ such that $A \cap B=\varnothing$. The reader is referred to [Ambrosio et al. 2000, Chapter 1] for further details of measures with values in finitedimensional inner-product spaces.

If $\boldsymbol{f}$ is a $Z$-valued map defined $\mathcal{L}^{1}$ almost everywhere in an interval $(0, \epsilon)$ where $\epsilon>0$, we say that $\boldsymbol{a} \in Z$ is an essential limit of $\boldsymbol{f}$ at 0 and write

$$
\boldsymbol{a}=\underset{\rho \rightarrow 0}{\operatorname{ess} \lim } \boldsymbol{f}(\rho)
$$

if there exists an $\mathcal{L}^{1}$ null set $N \subset(0, \epsilon)$ such that

$$
\boldsymbol{a}=\lim _{\substack{\rho \rightarrow 0 \\ \rho \in(0, \epsilon) \sim N}} \boldsymbol{f}(\rho),
$$

where the last limit is the ordinary limit relative to a subset of $(0, \epsilon)$. Note that, unlike the set $N$, the value $\boldsymbol{a}$ is uniquely determined, which justifies the notation (12).

\section{Maps of bounded variation, sets of finite perimeter, and admissible domains}

We state some basic definitions and properties of the space $B V$ of maps of bounded variation, of the space $S B V$ of special maps of bounded variation, of sets of finite perimeter, and of admissible domains that will be needed in the sequel. For more details, see [Ambrosio et al. 2000; Evans and Gariepy 1992; Ziemer 1989; Federer 1969].

Definition 3.1. We denote by $B V\left(\Omega, \mathrm{R}^{m}\right)$ the set of all $g \in L^{1}\left(\Omega, \mathrm{R}^{m}\right)$ such that there exists a measure $\mathrm{D} \boldsymbol{g} \in \mathcal{M}\left(\Omega, \mathrm{M}^{m \times n}\right)$ satisfying

$$
\int_{\Omega} \boldsymbol{g} \cdot \operatorname{div} \boldsymbol{T} d \mathcal{L}^{n}=-\int_{\Omega} \boldsymbol{T} \cdot d \mathrm{D} \boldsymbol{g}
$$

for each $\boldsymbol{T} \in C_{0}^{\infty}\left(\Omega, \mathrm{R}^{m \times n}\right)$. Here $\operatorname{div} \boldsymbol{T}$ is an $\mathrm{R}^{m}$-valued map on $\Omega$ such that

$$
\boldsymbol{a} \cdot \operatorname{div} \boldsymbol{T}=\operatorname{tr}\left(\mathrm{D}\left(\boldsymbol{T}^{\mathrm{T}} \boldsymbol{a}\right)\right)
$$

for each $\boldsymbol{a} \in \mathrm{R}^{m}$, where $\mathrm{D}\left(\boldsymbol{T}^{\mathrm{T}} \boldsymbol{a}\right)$ denotes the classical derivative of the map $\boldsymbol{T}^{\mathrm{T}} \boldsymbol{a}$. The elements of $B V\left(\Omega, \mathrm{R}^{m}\right)$ are called maps of bounded variation; the measure $\mathrm{D} g$ is uniquely determined by $\boldsymbol{g}$ and is called the weak (or generalized) derivative of $\boldsymbol{g}$. We denote by $\mathrm{M}(\mathrm{D} \boldsymbol{g})$ the mass of the measure $\mathrm{D} \boldsymbol{g}$ as defined in Section 2 and call $\mathrm{M}(\mathrm{D} \boldsymbol{g})$ the total variation of $\boldsymbol{g}$. Equations (11) and (13) provide

$$
\mathrm{M}(\mathrm{D} \boldsymbol{g})=\sup \left\{\int_{\Omega} \boldsymbol{g} \cdot \operatorname{div} \boldsymbol{T} d \mathcal{L}^{n}: \boldsymbol{T} \in C_{0}^{\infty}\left(\Omega, \mathrm{R}^{m \times n}\right),|\boldsymbol{T}| \leq 1 \text { on } \Omega\right\} .
$$


The choice of $\boldsymbol{T}$ represented by a matrix function with only the $(i, j)$ element different from 0 , where $i \in\{1, \ldots, m\}$ and $j \in\{1, \ldots, n\}$, reduces (13) to the usual index definition of $B V$ as in, e.g., [Ambrosio et al. 2000, Equation (3.2)].

The set $B V\left(\Omega, \mathrm{R}^{m}\right)$ is a Banach space under the norm

$$
|\boldsymbol{g}|_{B V\left(\Omega, \mathrm{R}^{m}\right)}:=|\boldsymbol{g}|_{L^{1}\left(\Omega, \mathrm{R}^{m}\right)}+\mathrm{M}(\mathrm{D} \boldsymbol{g}) .
$$

Definition 3.2. Let $\boldsymbol{g} \in L^{1}\left(\Omega, \mathrm{R}^{m}\right)$. We say that $\boldsymbol{g}$ has an approximate limit at $\boldsymbol{x} \in \Omega$ if there exists $\boldsymbol{a} \in \mathrm{R}^{m}$ such that

$$
\lim _{\rho \rightarrow 0} \kappa_{n}^{-1} \rho^{-n} \int_{\mathbf{B}(\boldsymbol{x}, \rho)}|\boldsymbol{g}-\boldsymbol{a}| d \mathcal{L}^{n}=0 .
$$

The value $\boldsymbol{a}$ is uniquely determined and is called the approximate limit of $\boldsymbol{g}$ at $\boldsymbol{x}$. The complement $S(\boldsymbol{g}) \subset \Omega$ in $\Omega$ of the set of all $\boldsymbol{x} \in \Omega$ where the approximate limit of $\boldsymbol{g}$ exists is called the approximate discontinuity set of $\boldsymbol{g}$.

Definition 3.3. Let $\boldsymbol{g} \in L^{1}\left(\Omega, \mathrm{R}^{m}\right)$. We say that $\boldsymbol{x} \in \Omega$ is an approximate jump point of $\boldsymbol{g}$ if there exist $\boldsymbol{a}, \boldsymbol{b} \in \mathrm{R}^{m}, \boldsymbol{a} \neq \boldsymbol{b}$, and $\boldsymbol{n} \in \mathrm{R}^{n}$ with $|\boldsymbol{n}|=1$ such that

$$
\begin{aligned}
& \lim _{\rho \rightarrow 0} \kappa_{n}^{-1} \rho^{-n} \int_{\mathbf{B}^{+}(\boldsymbol{x}, \rho, \boldsymbol{n})}|\boldsymbol{g}-\boldsymbol{a}| d \mathcal{L}^{n}=0, \\
& \lim _{\rho \rightarrow 0} \kappa_{n}^{-1} \rho^{-n} \int_{\mathbf{B}^{-}(\boldsymbol{x}, \rho, \boldsymbol{n})}|\boldsymbol{g}-\boldsymbol{b}| d \mathcal{L}^{n}=0 .
\end{aligned}
$$

Here

$$
\mathbf{B}^{ \pm}(\boldsymbol{x}, \rho, \boldsymbol{n})=\{\boldsymbol{y} \in \mathbf{B}(\boldsymbol{x}, \rho), \pm(\boldsymbol{y}-\boldsymbol{x}) \cdot \boldsymbol{n}>0\} .
$$

The triplet $(\boldsymbol{a}, \boldsymbol{b}, \boldsymbol{n})$, if it exists, is uniquely determined to within the interchange of $\boldsymbol{a}$ and $\boldsymbol{b}$ and a simultaneous change of the sign of $\boldsymbol{n}$. In any case, the product

$$
[g] \otimes n,
$$

occurring frequently below, is uniquely determined, where

$$
[g]=a-b
$$

is the jump of $\boldsymbol{g}$ at $\boldsymbol{x}$. We denote by $J(\boldsymbol{g})$ the set of all approximate jump points of $\boldsymbol{g}$ and call any $\pm \boldsymbol{n}$ the normal of $J(\boldsymbol{g})$ at $\boldsymbol{x}$.

The following result describes the relationship between the sets $S(g)$ and $J(g)$ :

Theorem 3.4. If $\boldsymbol{g} \in B V\left(\Omega, \mathrm{R}^{m}\right)$, then:

(i) $J(\boldsymbol{g}) \subset S(\mathrm{~g})$ and $\mathcal{H}^{n-1}(S(\boldsymbol{g}) \sim J(\mathrm{~g}))=0$.

(ii) $J(\mathrm{~g})$ is countably $\left(\mathcal{H}^{n-1}, n-1\right)$-rectifiable in the sense that $\mathcal{H}^{n-1}$ almost all of $\boldsymbol{J}(\boldsymbol{g})$ can be covered by countably many class -1 surfaces $C_{k}, k=1, \ldots$ of dimension $n-1$ in $\mathrm{R}^{n}$. 
The derivative of a map of bounded variation has the following well known structure. The subsequent treatment uses especially the jump and the absolutely continuous parts of the derivative to be introduced now.

Theorem 3.5. If $\boldsymbol{g} \in B V\left(\Omega, \mathrm{R}^{m}\right)$, then:

(i) The derivative $\mathrm{Dg}$ has a unique decomposition

$$
\mathrm{D} \boldsymbol{g}=\mathrm{D}_{\mathrm{a}} \boldsymbol{g} \mathcal{L}^{n}\left\llcorner\Omega+\mathrm{D}_{\mathrm{c}} \boldsymbol{g}+\mathrm{D}_{\mathrm{j}} \boldsymbol{g},\right.
$$

where $\mathrm{D}_{\mathrm{a}} \mathbf{g}$, the absolutely continuous part of $\mathrm{D} \boldsymbol{g}$, is a map in $L^{1}\left(\Omega, \mathrm{M}^{m \times n}\right)$; $\mathrm{D}_{\mathrm{c}} \boldsymbol{g}$, the Cantor part of $\mathrm{D} \boldsymbol{g}$, is a measure on $\Omega$ singular with respect to $\mathcal{L}^{n}$ and diffuse with respect to $\mathcal{H}^{n-1}$, i.e., $\mathrm{D}_{\mathrm{c}} \mathrm{g}$ is supported by a set of null Lebesgue measure in $\mathrm{R}^{n}$ and $\mathrm{D}_{\mathrm{c}} \boldsymbol{g}(B)=\mathbf{0}$ for each Borel subset $B$ of $\Omega$ of finite $\mathcal{H}^{n-1}$ measure; and $\mathrm{D}_{\mathrm{j}} \boldsymbol{g}$, the jump part of $\mathrm{D} \boldsymbol{g}$, is a measure absolutely continuous with respect to $\mathcal{H}^{n-1}$.

(ii) The jump part $\mathrm{D}_{\mathrm{j}} \mathrm{g}$ is supported by $\mathrm{J}(\mathrm{g})$, and in fact,

$$
\mathrm{D}_{\mathrm{j}} \boldsymbol{g}=[\boldsymbol{g}] \otimes \boldsymbol{n} \mathcal{H}^{n-1}\llcorner J(\boldsymbol{g}),
$$

where, for every point $\boldsymbol{x}$ of $\boldsymbol{J}(\boldsymbol{g})$, the value $[\boldsymbol{g}] \otimes \boldsymbol{n}$ is the product (15).

(iii) For $\mathcal{L}^{n}$ almost every point $\boldsymbol{x}$ of $\Omega$, we have

$$
\mathrm{D}_{\mathrm{a}} \boldsymbol{g}(\boldsymbol{x})=\lim _{\rho \rightarrow 0} \kappa_{n}^{-1} \rho^{-n} \mathrm{D}(\mathbf{B}(\boldsymbol{x}, \rho)) .
$$

Definition 3.6. We denote by $\operatorname{SBV}\left(\Omega, \mathrm{R}^{m}\right)$ the set of all $\boldsymbol{g} \in B V\left(\Omega, \mathrm{R}^{m}\right)$ with $\mathrm{D}_{\mathrm{c}} \boldsymbol{g}=\mathbf{0}$. The elements of $\operatorname{SBV}\left(\Omega, \mathrm{R}^{m}\right)$ are called special maps of bounded variation.

$\operatorname{SBV}\left(\Omega, \mathrm{R}^{m}\right)$ is a closed subspace of $B V\left(\Omega, \mathrm{R}^{m}\right)$ under the norm $|\cdot|_{B V\left(\Omega, \mathrm{R}^{m}\right)}$.

Theorem 3.7 [Alberti 1991]. If $\Omega$ is bounded, then for any $\boldsymbol{G} \in L^{1}\left(\Omega, \mathbf{M}^{m \times n}\right)$, there exists a $\boldsymbol{g} \in \operatorname{SBV}\left(\Omega, \mathrm{R}^{m}\right)$ such that $\mathrm{D}_{\mathrm{a}} \boldsymbol{g}=\boldsymbol{G}$; moreover, there exists a constant $c \in \mathrm{R}$ depending only on $\Omega$ such that the map $\mathrm{g}$ as above can be chosen to satisfy

$$
\mathrm{M}(\boldsymbol{g}) \leq c|\boldsymbol{G}|_{L^{1}\left(\Omega, \mathrm{M}^{m \times n}\right)} .
$$

We conclude this section with basic information on sets of finite perimeter and on admissible domains. Sets of finite perimeter fall in the framework of $B V$ as will be explained below. For a subset of the class of sets of finite perimeter called admissible domains (see below), we shall establish the approximation theorem. The distinguishing feature of admissible domains $\Omega$ is that maps from $B V\left(\Omega, \mathrm{R}^{m}\right)$ have well defined boundary values.

Definition 3.8. A set $E \subset \mathrm{R}^{n}$ is said to have a finite perimeter if $1_{E} \in B V\left(\mathrm{R}^{n}, \mathrm{R}\right)$, where $1_{E}$ denotes the characteristic function of $E$. The perimeter of $E$ is $\mathrm{M}\left(\mathrm{D} 1_{E}\right)$. 
The measure-theoretic boundary of $E$ is the set $S\left(1_{E}\right)$ that differs from the reduced boundary $\operatorname{bdry}_{*}(E):=J\left(1_{E}\right)$ by a set of $\mathcal{H}^{n-1}$ measure 0 .

Theorem 3.9. If $E$ is a set of finite perimeter, then for every $\boldsymbol{x} \in \operatorname{bdry}_{*}(E)$, the triplet $(\boldsymbol{a}, \boldsymbol{b}, \boldsymbol{n})$ as in Definition 3.3 can be chosen to be $(0,1, \boldsymbol{n}(\boldsymbol{x}))$; with this choice, $\boldsymbol{n}(\boldsymbol{x})$ is uniquely determined and is called the measure-theoretic normal to $E$ at $\boldsymbol{x}$. Equations (14) then imply the following well known formulas:

$$
\begin{gathered}
\lim _{\rho \rightarrow 0} \kappa_{n}^{-1} \rho^{-n} \mathcal{L}^{n}\left(E \cap \mathbf{B}^{+}(\boldsymbol{x}, \rho, \boldsymbol{n})\right)=0, \\
\left.\lim _{\rho \rightarrow 0} \kappa_{n}^{-1} \rho^{-n} \mathcal{L}^{n}\left(\mathbf{B}^{-}(\boldsymbol{x}, \rho, \boldsymbol{n}) \sim E\right)\right)=0,
\end{gathered}
$$

where $\boldsymbol{n}=\boldsymbol{n}(\boldsymbol{x})$. One has

$$
\mathrm{D} 1_{E}=\boldsymbol{n} \mathcal{H}^{n-1} \mathrm{Lbdry}_{*} E .
$$

Thus, even $1_{E} \in S B V\left(\mathrm{R}^{n}, \mathrm{R}\right)$.

Definition 3.10 [Ziemer 1989, Definition 5.10.1]. A bounded open set $\Omega \subset \mathrm{R}^{n}$ is said to be an admissible domain if it has a finite perimeter and the following two conditions are satisfied:

(i) $\mathcal{H}^{n-1}$ (bdry $B \sim$ bdry $\left._{*} B\right)=0$.

(ii) There exists a constant $M$, and for each $\boldsymbol{x} \in$ bdry $\Omega$, there is a ball $\mathbf{B}(\boldsymbol{x}, r)$ with

$$
\mathcal{H}^{n-1}\left(\operatorname{bdry}_{*} E \cap \operatorname{bdry}_{*} \Omega\right) \leq M \mathcal{H}^{n-1}\left(\Omega \cap \text { bdry }_{*} E\right)
$$

whenever $E \subset \operatorname{cl} \Omega \cap \mathbf{B}(\boldsymbol{x}, r)$ is a set of finite perimeter.

Each open bounded set with lipschitzian boundary is an admissible domain [Ziemer 1989, Remark 5.10.2]. The following two theorems describe the main virtues of admissible domains:

Theorem 3.11 (see [Ziemer 1989, Section 5.10]). If $\Omega$ is an admissible domain and $\boldsymbol{g} \in B V\left(\Omega, \mathrm{R}^{m}\right)$, then there exist an $\mathcal{H}^{n-1}$-measurable map $\boldsymbol{g}_{\text {bdry } \Omega}$ on bdry $\Omega$ such that

$$
\int_{\Omega} \boldsymbol{g} \cdot \operatorname{div} \boldsymbol{T} d \mathcal{L}^{n}+\int_{\Omega} \boldsymbol{T} \cdot d \mathrm{D} \boldsymbol{g}=\int_{\mathrm{bdry}(\Omega)} \boldsymbol{T} \boldsymbol{n} \cdot \boldsymbol{g}_{\mathrm{bdry} \Omega} d \mathcal{H}^{n-1}
$$

for every class -1 map $\boldsymbol{T}$ on $\Omega$ with values in $\mathrm{M}^{m \times n}$ that has a continuous extension (again denoted by $\boldsymbol{T}$ ) to $\mathrm{cl} \Omega$, where $\boldsymbol{n}$ is the measure-theoretic normal to $\Omega$. There exists a $c \in \mathrm{R}$ depending only on $\Omega$ such that

$$
\int_{\text {bdry } \Omega}\left|\boldsymbol{g}_{\text {bdry } \Omega}\right| d \mathcal{H}^{n-1} \leq c|\boldsymbol{g}|_{B V\left(\Omega, \mathrm{R}^{m}\right)}
$$


The map $\boldsymbol{g}_{\mathrm{bdry} \Omega}$ is determined to within a change on a set of $\mathcal{H}^{n-1}$-measure 0 and is called the trace of $\mathbf{g}$. One has

$$
\lim _{r \rightarrow 0} \kappa_{n}^{-1} r^{-n} \int_{\mathbf{B}(\boldsymbol{y}, r) \cap \Omega}\left|\boldsymbol{g}-\boldsymbol{g}_{\text {bdry } \Omega}(\boldsymbol{y})\right| d \mathcal{L}^{n}=0
$$

for $\mathcal{H}^{n-1}$ almost every point $\boldsymbol{y}$ of bdry $\Omega$.

Theorem 3.12 (cf. [Ziemer 1989, Lemma 5.10.4]). If $\Omega$ is an admissible domain and $\boldsymbol{g} \in B V\left(\Omega, \mathrm{R}^{m}\right)$, then the extension $\boldsymbol{g}_{0}$ of $\boldsymbol{g}$ to $\mathrm{R}^{n}$ equal to 0 outside $\Omega$ satisfies $\boldsymbol{g}_{0} \in B V\left(\mathrm{R}^{n}, \mathrm{R}^{m}\right)$,

$$
\mathrm{D} \boldsymbol{g}_{0}=\mathrm{D} \boldsymbol{g}-\boldsymbol{g}_{\text {bdry } \Omega} \otimes \boldsymbol{n} \mathcal{H}^{n-1}\llcorner\text { bdry } \Omega,
$$

and there exists a $c \in \mathrm{R}$ depending only on $\Omega$ such that

$$
\left|\boldsymbol{g}_{0}\right|_{B V\left(\mathrm{R}^{n}, \mathrm{R}^{m}\right)} \leq c|\boldsymbol{g}|_{B V\left(\Omega, \mathrm{R}^{m}\right)} .
$$

\section{The $B V$ setting of structured deformations}

For the purpose of the approximation theorem and the limit relation (as stated in Section 1), we enlarge the set $\operatorname{SBV}\left(\Omega, \mathrm{R}^{m}\right) \times L^{1}\left(\Omega, \mathrm{M}^{m \times n}\right)$ of structured deformations of Choksi and Fonseca [1997] to form the set $B V\left(\Omega, \mathrm{R}^{m}\right) \times L^{1}\left(\Omega, \mathrm{M}^{m \times n}\right)$. We furthermore interpret the elements $g \in S B V\left(\Omega, \mathrm{R}^{m}\right)$ as the macroscopic deformations of the body $\Omega$ with macroscopic crack site $J(\boldsymbol{g})$. We note that the space of structured deformations $(\mathcal{K}, \boldsymbol{g}, \boldsymbol{G})$ of Del Piero and Owen [1993] as described in Section 1 with $\mathcal{K}=\varnothing$ is a subset of $S B V\left(\Omega, \mathrm{R}^{m}\right) \times L^{1}\left(\Omega, \mathrm{M}^{m \times n}\right) \subset$ $B V\left(\Omega, \mathrm{R}^{m}\right) \times L^{1}\left(\Omega, \mathrm{M}^{m \times n}\right)$. In a general, $(\boldsymbol{g}, \boldsymbol{G}) \in B V\left(\Omega, \mathrm{R}^{m}\right) \times L^{1}\left(\Omega, \mathrm{M}^{m \times n}\right)$, the map $\boldsymbol{g}$ is the possibly discontinuous macroscopic displacement, of the body $\Omega$ and $\boldsymbol{G}$ is a microscopic disarrangement as explained in the introduction and in accord with the original papers by Del Piero and Owen [1993; 1995].

\section{Proof of the approximation theorem}

The proof of the Approximation Theorem is based on the decomposition of $\mathrm{R}^{n}$ into the disjoint union of sufficiently small cubes of equal edge length and with faces parallel to the natural coordinate planes in $\mathrm{R}^{n}$. Various maps involved in the construction are then approximated by (generally) discontinuous maps constant on the cubes (as in the present section) or by discontinuous maps linear on the cubes (as in the Appendix below).

For each positive integer $k$, consider the decomposition of $\mathrm{R}^{n}$ into the system of cubes

$$
C(k, \boldsymbol{p}):=C / k+\boldsymbol{p}, \quad \boldsymbol{p} \in \mathrm{Z}^{n} / k,
$$


where $C:=[0,1)^{n}, C / k:=\{\boldsymbol{x} / k: \boldsymbol{x} \in C\}, Z^{n}$ is the set of $n$ tuples of integers, and $\mathrm{Z}^{n} / k:=\left\{z / k: z \in \mathrm{Z}^{n}\right\}$.

Let $\boldsymbol{e}_{1}, \ldots, \boldsymbol{e}_{n}$ be the natural orthonormal basis in $\mathrm{R}^{n}$.

Lemma 5.1. Let $k$ be a positive integer, let $\boldsymbol{f} \in C_{0}^{\infty}\left(\mathrm{R}^{n}, \mathrm{R}^{m}\right)$, and let $\boldsymbol{p}, \boldsymbol{q} \in \mathrm{Z}^{n} / k$ be such that $P:=C(k, \boldsymbol{p})$ and $Q:=C(k, \boldsymbol{q})$ are two adjacent cubes sharing the common face $F:=\operatorname{cl} P \cap \operatorname{cl} Q \neq \varnothing$ of normal $n$ pointing from $P$ to $Q$. Let $\boldsymbol{m}: P \cup Q \rightarrow \mathrm{R}^{m}$ be defined by

$$
\boldsymbol{m}(\boldsymbol{x})= \begin{cases}\boldsymbol{a} & \text { if } \boldsymbol{x} \in P \\ \boldsymbol{b} & \text { if } \boldsymbol{x} \in Q\end{cases}
$$

where

$$
\boldsymbol{a}=k^{n} \int_{P} \boldsymbol{f} d \mathcal{L}^{n} \quad \text { and } \quad \boldsymbol{b}=k^{n} \int_{Q} \boldsymbol{f} d \mathcal{L}^{n}
$$

are the averages of $\boldsymbol{f}$ over the two cubes. Then $\boldsymbol{m} \in S B V\left(\operatorname{int}(P \cup Q), \mathrm{R}^{m}\right)$,

$$
\mathrm{D} \boldsymbol{m}=(\boldsymbol{b}-\boldsymbol{a}) \otimes \boldsymbol{n} \mathcal{H}^{n-1}\left\llcorner F, \quad \mathrm{D}_{\mathrm{a}} \boldsymbol{m}=\mathbf{0},\right.
$$

and

$$
\mathrm{M}(\mathrm{D} \boldsymbol{m}) \leq \int_{P \cup Q}\left|\mathrm{D}_{\boldsymbol{n}} \boldsymbol{f}\right| d \mathcal{L}^{n},
$$

where $\mathrm{D}_{\boldsymbol{n}} \boldsymbol{f}$ is the directional derivative of $\boldsymbol{f}$ in the direction $\boldsymbol{n}$.

Proof. We only prove (21) since the other assertions of the lemma are immediate. Let $\boldsymbol{x} \in P$ be arbitrary, and denote $\boldsymbol{y}(\boldsymbol{x}):=\boldsymbol{x}+\boldsymbol{n} / k$ so that $\boldsymbol{y}(\boldsymbol{x}) \in Q$. Then

$$
\boldsymbol{f}(\boldsymbol{y}(\boldsymbol{x}))-\boldsymbol{f}(\boldsymbol{x})=k^{-1} \int_{0}^{1} \mathrm{D}_{\boldsymbol{n}} \boldsymbol{f}(\boldsymbol{x}+t \boldsymbol{n}) d t,
$$

and hence,

$$
|\boldsymbol{f}(\boldsymbol{y}(\boldsymbol{x}))-\boldsymbol{f}(\boldsymbol{x})| \leq k^{-1} \int_{0}^{1}\left|\mathrm{D}_{\boldsymbol{n}} \boldsymbol{f}(\boldsymbol{x}+t \boldsymbol{n})\right| d t .
$$

We have

$$
\begin{aligned}
U:=\left|\int_{Q} \boldsymbol{f} d \mathcal{L}^{n}-\int_{P} \boldsymbol{f} d \mathcal{L}^{n}\right| & =\left|\int_{P} \boldsymbol{f}(\boldsymbol{y}(\boldsymbol{x})) d \mathcal{L}^{n}(\boldsymbol{x})-\int_{P} \boldsymbol{f}(\boldsymbol{x}) d \mathcal{L}^{n}(\boldsymbol{x})\right| \\
& \leq \int_{P}|\boldsymbol{f}(\boldsymbol{y}(\boldsymbol{x}))-\boldsymbol{f}(\boldsymbol{x})| d \mathcal{L}^{n}(\boldsymbol{x}) .
\end{aligned}
$$

Consequently, integrating (22) over $P$, we obtain 


$$
\begin{aligned}
U \leq k^{-1} \int_{0}^{1} \int_{P} \mid \mathrm{D}_{\boldsymbol{n}} \boldsymbol{f}(\boldsymbol{x}+\text { tn }) \mid d \mathcal{L}^{n}(\boldsymbol{x}) d t & =k^{-1} \int_{0}^{1} \int_{P+t \boldsymbol{n}}\left|\mathrm{D}_{\boldsymbol{n}} \boldsymbol{f}\right| d \mathcal{L}^{n} d t \\
& \leq k^{-1} \int_{0}^{1} \int_{P \cup Q}\left|\mathrm{D}_{\boldsymbol{n}} \boldsymbol{f}\right| d \mathcal{L}^{n} d t \\
& =k^{-1} \int_{P \cup Q}\left|\mathrm{D}_{\boldsymbol{n}} \boldsymbol{f}\right| d \mathcal{L}^{n} ;
\end{aligned}
$$

the last inequality above follows from $P+t \boldsymbol{n} \subset P \cup Q$ for each $t \in[0,1]$. Multiplying the just proved inequality

$$
\left|\int_{Q} \boldsymbol{f} d \mathcal{L}^{n}-\int_{P} \boldsymbol{f} d \mathcal{L}^{n}\right| \leq k^{-1} V, \quad V:=\int_{P \cup Q}\left|\mathrm{D}_{\boldsymbol{n}} \boldsymbol{f}\right| d \mathcal{L}^{n},
$$

by $k^{n}$, we obtain

$$
|\boldsymbol{b}-\boldsymbol{a}| \leq k^{n-1} V
$$

and a combination with $(20)_{1}$ provides that the total variation (measure) $|\mathrm{Dm}|$ satisfies

$$
|\mathrm{D} \boldsymbol{m}|=|\boldsymbol{b}-\boldsymbol{a}| \mathcal{H}^{n-1}\left\llcorner F \leq k^{n-1} V \mathcal{H}^{n-1}\llcorner F .\right.
$$

Integrating over $\mathrm{R}^{n}$, we obtain

$$
\mathrm{M}(\mathrm{D} \boldsymbol{m})=|\mathrm{D} \boldsymbol{m}|\left(\mathrm{R}^{n}\right) \leq k^{n-1} V \mathcal{H}^{n-1}(F)=V,
$$

which is (21).

Proposition 5.2. Let $\boldsymbol{f} \in C_{0}^{\infty}\left(\mathrm{R}^{n}, \mathrm{R}^{m}\right)$. There exists a sequence $\boldsymbol{m}_{k} \in S B V\left(\mathrm{R}^{n}, \mathrm{R}^{m}\right)$ such that

$$
\begin{aligned}
& \boldsymbol{m}_{k} \rightarrow \boldsymbol{f} \quad \text { in } L^{1}\left(\mathrm{R}^{n}, \mathrm{R}^{m}\right), \\
& \mathrm{D}_{\mathrm{a}} \boldsymbol{m}_{k}=\mathbf{0} \quad \text { on } \mathrm{R}^{n} \text { for all } k=1, \ldots,
\end{aligned}
$$

and

$$
\mathrm{M}\left(\mathrm{Dm}_{k}\right) \leq 2 n \int_{\mathrm{R}^{n}}|\mathrm{D} \boldsymbol{f}| d \mathcal{L}^{n} .
$$

Proof. For each positive integer $k$, consider the decomposition of $\mathrm{R}^{n}$ into the system of cells as in (18). Let $\boldsymbol{m}_{k}: \mathrm{R}^{n} \rightarrow \mathrm{R}^{n}$ be defined by

$$
\boldsymbol{m}_{k}(\boldsymbol{x})=\boldsymbol{f}(k, \boldsymbol{p})
$$

for each $\boldsymbol{x} \in \mathrm{R}^{n}$, where $\boldsymbol{p} \in \mathrm{Z}^{n} / k$ is uniquely determined by the requirement that $\boldsymbol{x} \in C(k, \boldsymbol{p})$ and where

$$
\boldsymbol{f}(k, \boldsymbol{p})=k^{n} \int_{C(k, \boldsymbol{p})} \boldsymbol{f} d \mathcal{L}^{n}
$$


Then $\boldsymbol{m}_{k}$ is piecewise constant, with all points of jump discontinuity contained in the union

$$
\bigcup_{i=1}^{n} \bigcup_{l \in \mathrm{Z} / k} P_{k, i, l},
$$

where

$$
P_{k, i, l}=\left\{\boldsymbol{x} \in \mathrm{R}^{n}: \boldsymbol{x} \cdot \boldsymbol{e}_{i}=l\right\}
$$

for any $l \in \mathrm{Z} / k$. Here for each $i=1, \ldots, n$, the system

$$
\mathcal{S}_{k, i}=\left\{P_{k, i, l}: l \in \mathrm{Z} / k\right\}
$$

forms an equidistant system of parallel planes perpendicular to $\boldsymbol{e}_{i}$.

We now fix $k=1, \ldots$ and $i=1, \ldots, n$ and denote by $S_{k, i} \subset \mathrm{R}^{n}$ the union of the system $\mathcal{S}_{k, i}$ of planes perpendicular to $\boldsymbol{e}_{i}$. Next we apply Lemma 5.1 to each pair of adjacent cubes $C(k, \boldsymbol{p})$ and $C(k, \boldsymbol{q})$ with $\boldsymbol{p}, \boldsymbol{q} \in \mathrm{Z}^{n} / k$ sharing a common face perpendicular to $\boldsymbol{e}_{i}$. Summing the inequality (21) over all such pairs, we obtain

$$
\mathrm{M}\left(\mathrm{D} \boldsymbol{m}_{k}\left\llcorner S_{k, i}\right) \leq 2 \int_{\mathrm{R}^{n}}\left|\mathrm{D}_{\boldsymbol{e}_{i}} \boldsymbol{f}\right| d \mathcal{L}^{n},\right.
$$

where $\mathrm{D}_{\boldsymbol{e}_{i}} \boldsymbol{f}$ is the directional derivative of $\boldsymbol{f}$ in the direction $\boldsymbol{e}_{i}$. Summing over all $i$, we obtain (25). Relation (23) follows immediately from the well known properties of the piecewise-constant approximations on system of cubes of decreasing edge length. Finally (24) follows from the piecewise-constant character of $\boldsymbol{m}_{k}$.

Proof of the approximation theorem. By Alberti's theorem (Theorem 3.7), there exists $\boldsymbol{h} \in \operatorname{SBV}\left(\Omega, \mathrm{R}^{m}\right)$ such that

$$
\mathrm{D}_{\mathrm{a}} \boldsymbol{h}=\boldsymbol{G} \quad \text { on } \Omega .
$$

Put $\boldsymbol{l}:=\boldsymbol{g}-\boldsymbol{h}$, which is an element of $B V\left(\Omega, \mathrm{R}^{m}\right)$. Since $\Omega$ is an admissible domain, the extension $\boldsymbol{l}_{0}$ of $\boldsymbol{l}$ to $\mathrm{R}^{n}$ equal to $\mathbf{0}$ outside $\Omega$ satisfies $\boldsymbol{l}_{0} \in B V\left(\mathrm{R}^{n}, \mathrm{R}^{m}\right)$ by Theorem 3.12. Let $f_{k}$ be a sequence of mollifications of $\boldsymbol{l}_{0}$ on $\mathrm{R}^{n}$ with the mollification parameter tending to 0 so that $f_{k} \in C_{0}^{\infty}\left(\mathrm{R}^{n}, \mathrm{R}^{m}\right)$,

$$
\begin{aligned}
& \int_{\mathrm{R}^{n}}\left|\mathrm{D} \boldsymbol{f}_{k}\right| d \mathcal{L}^{n} \leq \mathrm{M}\left(\mathrm{D} \boldsymbol{l}_{0}\right), \\
& \boldsymbol{f}_{k} \rightarrow \boldsymbol{l}_{0} \text { in } L^{1}\left(\mathrm{R}^{n}, \mathrm{R}^{m}\right),
\end{aligned}
$$

and hence in particular

$$
\boldsymbol{f}_{k} \mid \Omega \rightarrow \boldsymbol{l} \text { in } L^{1}\left(\Omega, \mathrm{R}^{m}\right) .
$$


Applying Proposition 5.2 with $\boldsymbol{f}$ replaced by $\boldsymbol{f}_{k}$, we find that for each $k$ there exists an $\boldsymbol{m}_{k} \in S B V\left(\mathrm{R}^{n}, \mathrm{R}^{m}\right)$ such that

$$
\begin{gathered}
\left|\boldsymbol{m}_{k}-\boldsymbol{f}_{k}\right|_{L^{1}\left(\mathrm{R}^{n}, \mathrm{R}^{m}\right)}<1 / k, \\
\mathrm{D}_{\mathrm{a}} \boldsymbol{m}_{k}=\mathbf{0} \text { on } \mathrm{R}^{n},
\end{gathered}
$$

and

$$
\mathrm{M}\left(\mathrm{D} \boldsymbol{m}_{k}\right) \leq 2 n \int_{\mathrm{R}^{n}}\left|\mathrm{D} \boldsymbol{f}_{k}\right| d \mathcal{L}^{n}
$$

We put

$$
\boldsymbol{g}_{k}=\boldsymbol{m}_{k} \mid \Omega+\boldsymbol{h}
$$

for $k=1, \ldots$ so that $\boldsymbol{g}_{k} \in \operatorname{SBV}\left(\Omega, \mathrm{R}^{m}\right)$. Equations (29) and (30) imply

$$
\boldsymbol{m}_{k} \mid \Omega \rightarrow \boldsymbol{l} \text { in } L^{1}\left(\Omega, \mathrm{R}^{m}\right) \text { as } k \rightarrow \infty,
$$

and hence, we have (4) 1 . Further, (31) and (27) imply (4) 2 . Finally, (32), (28), and $\boldsymbol{h} \in \operatorname{SBV}\left(\Omega, \mathrm{R}^{m}\right)$ imply (5). Assertion (6) then follows by an easy argument that is left to the reader.

\section{Proof of the limit relation}

Lemma 6.1. Let $\Omega$ be bounded, let $\boldsymbol{g}$ be a map (not a class of equivalence) in $B V\left(\Omega, \mathrm{R}^{m}\right)$, let $\boldsymbol{x} \in \Omega$, and let $\epsilon>0$ be such that $\mathbf{B}(\boldsymbol{x}, \epsilon) \subset \Omega$. Then for $\mathcal{L}^{1}$ almost every $\rho \in(0, \epsilon), \boldsymbol{g} \mid$ bdry $\mathbf{B}(\boldsymbol{x}, \rho)$ is the trace of $\boldsymbol{g} \mid \mathbf{B}(\boldsymbol{x}, \rho) \in B V\left(\mathbf{B}\left(\boldsymbol{x}, \rho, \mathbf{R}^{m}\right)\right.$.

Proof. By the Lebesgue differentiation theorem, there exists a Borel set $E \subset \Omega$ with $\mathcal{L}^{n}(E)=0$ such that for every $\boldsymbol{y} \in \Omega \sim E$ we have

$$
\lim _{r \rightarrow 0} \kappa_{n}^{-1} r^{-n} \int_{\mathbf{B}(\boldsymbol{y}, r) \cap \Omega}|\boldsymbol{g}-\boldsymbol{g}(\boldsymbol{y})| d \mathcal{L}^{n}=0 .
$$

Since by Fubini's theorem

$$
0=\mathcal{L}^{n}(E)=\int_{0}^{\infty} \mathcal{H}^{n-1}(E \cap \operatorname{bdry} \mathbf{B}(\boldsymbol{x}, \rho)) d \mathcal{L}^{1}(\rho),
$$

we see that for $\mathcal{L}^{1}$ almost every $\rho>0$ we have

$$
\mathcal{H}^{n-1}(E \cap \operatorname{bdry} \mathbf{B}(\boldsymbol{x}, \rho))=0 .
$$

For every such a $\rho \in(0, \epsilon)$, we have (33) for $\mathcal{H}^{n-1}$ almost every $\boldsymbol{y} \in \operatorname{bdry} \mathbf{B}(\boldsymbol{x}, \rho)$ and hence in particular also

$$
\lim _{r \rightarrow 0} \kappa_{n}^{-1} r^{-n} \int_{\mathbf{B}(\boldsymbol{y}, r) \cap \mathbf{B}(\boldsymbol{x}, \rho)}|\boldsymbol{g}-\boldsymbol{g}(\boldsymbol{y})| d \mathcal{L}^{n}=0
$$


since $\mathbf{B}(\boldsymbol{x}, \rho) \subset \Omega$. A comparison with (17) of Theorem 3.11 written for $\Omega$ replaced by $\mathbf{B}(\boldsymbol{x}, \rho)$ shows that $\boldsymbol{g}(\boldsymbol{y})$ coincides with the trace of $\boldsymbol{g} \mid \mathbf{B}(\boldsymbol{x}, \rho)$ for $\mathcal{H}^{n-1}$ almost every $\boldsymbol{y} \in \operatorname{bdry} \mathbf{B}(\boldsymbol{x}, \rho)$.

Proof of the limit relation. Let us extend $\boldsymbol{g}$ and $\boldsymbol{g}_{k}$ by $\mathbf{0}$ outside $\Omega$. We first note that by $(7)_{1}$ we may pass to a subsequence of $\boldsymbol{g}_{k}$ (not relabeled) such that $\left|\boldsymbol{g}-\boldsymbol{g}_{k}\right|_{L^{1}\left(\Omega, \mathrm{R}^{m}\right)}<2^{-k}$ so that the function

$$
\varphi(\boldsymbol{x})=\sum_{k=1}^{\infty}\left|\boldsymbol{g}(\boldsymbol{x})-\boldsymbol{g}_{k}(\boldsymbol{x})\right|
$$

satisfies

$$
\int_{\Omega} \varphi d \mathcal{L}^{n} \leq 1
$$

Let $\boldsymbol{x} \in \Omega$ be fixed, and let $\epsilon>0$ be any number satisfying $\mathbf{B}(\boldsymbol{x}, \epsilon) \subset \Omega$. Since

$$
\int_{0}^{\infty} \int_{\operatorname{bdry} \mathbf{B}(x, \rho)} \varphi d \mathcal{H}^{n-1} d \rho=\int_{\Omega} \varphi d \mathcal{L}^{n} \leq 1
$$

by (34), there exists a subset $N_{1}$ of $(0, \epsilon)$ with $\mathcal{L}^{1}\left(N_{1}\right)=0$ such that

$$
\int_{\operatorname{bdry} \mathbf{B}(\boldsymbol{x}, \rho)} \varphi d \mathcal{H}^{n-1} \equiv \sum_{k=1}^{\infty} \int_{\operatorname{bdry} \mathbf{B}(\boldsymbol{x}, \rho)}\left|\boldsymbol{g}-\boldsymbol{g}_{k}\right| d \mathcal{H}^{n-1}<\infty
$$

for every $\rho \in(0, \epsilon) \sim N_{1}$. Hence, for every $\rho \in(0, \epsilon) \sim N_{1}$, we have

$$
\int_{\operatorname{bdry} \mathbf{B}(x, \rho)}\left|\boldsymbol{g}-\boldsymbol{g}_{k}\right| d \mathcal{H}^{n-1} \rightarrow 0
$$

and hence

$$
g_{k} \rightarrow \boldsymbol{g}
$$

in the Lebesgue space $L^{1}$ (bdry $\left.\mathbf{B}(\boldsymbol{x}, \rho), \mathcal{H}^{n-1}\right)$ on bdry $\mathbf{B}(\boldsymbol{x}, \rho)$ relative to the measure $\mathcal{H}^{n-1}$. By Lemma 6.1 , for every $k=1, \ldots$, there exists a subset $M_{k}$ of $(0, \epsilon)$ with $\mathcal{L}^{1}\left(M_{k}\right)=0$ such that for every $\rho \in(0, \epsilon) \sim M_{k}$ the restriction of the map $\boldsymbol{g}_{k} \mid$ bdry $\mathbf{B}(\boldsymbol{x}, \rho)$ is the trace of $\boldsymbol{g}_{k} \mid \mathbf{B}(\boldsymbol{x}, \rho) \in B V\left(\mathbf{B}(\boldsymbol{x}, \rho), \mathrm{R}^{m}\right)$. Let

$$
N=N_{1} \cup \bigcup_{k=1}^{\infty} M_{k}
$$

so that $\mathcal{L}^{1}(N)=0$. For every $\rho \in(0, \epsilon) \sim N$, we have

$$
\int_{\mathrm{bdry} \mathbf{B}(\boldsymbol{x}, \rho)} \varphi \boldsymbol{g}_{k} \otimes \boldsymbol{n} d \mathcal{H}^{n-1}=\int_{\mathbf{B}(\boldsymbol{x}, \rho)} \boldsymbol{g}_{k} \otimes \mathrm{D} \varphi d \mathcal{L}^{n}+\int_{\mathbf{B}(\boldsymbol{x}, \rho)} \varphi d \mathrm{D} \boldsymbol{g}_{k}
$$


for all $k=1, \ldots$ and for any $\varphi \in C_{0}^{\infty}\left(\mathrm{R}^{n}\right)$ where $\boldsymbol{n}$ is the normal to $\mathbf{B}(\boldsymbol{x}, \rho)$. The limit using (36), (7) ${ }_{1}$, and (6) then gives

$$
\int_{\operatorname{bdry} \mathbf{B}(\boldsymbol{x}, \rho)} \varphi \boldsymbol{g} \otimes \boldsymbol{n} d \mathcal{H}^{n-1}=\int_{\mathbf{B}(\boldsymbol{x}, \rho)} \boldsymbol{g} \otimes \mathrm{D} \varphi d \mathcal{L}^{n}+\int_{\mathbf{B}(\boldsymbol{x}, \rho)} \varphi d \mathrm{D} \boldsymbol{g},
$$

and hence, $\boldsymbol{g} \mid$ bdry $\mathbf{B}(\boldsymbol{x}, \rho)$ is the trace of $\boldsymbol{g} \mid \mathbf{B}(\boldsymbol{x}, \rho) \in B V\left(\mathbf{B}(\boldsymbol{x}, \rho), \mathrm{R}^{m}\right)$ for every $\rho \in(0, \epsilon) \sim N$. In particular, for $\varphi \equiv 1$ on $\mathrm{R}^{n}$, we obtain from (37) and (38)

$$
\mathrm{Dg}_{k}(\mathbf{B}(\boldsymbol{x}, \rho)) \rightarrow \int_{\operatorname{bdry} \mathbf{B}(\boldsymbol{x}, \rho)} \boldsymbol{g} \otimes \boldsymbol{n} d \mathcal{H}^{n-1}=\mathrm{D} \boldsymbol{g}(\mathbf{B}(\boldsymbol{x}, \rho)),
$$

i.e.,

$$
\operatorname{Dg}_{k}(\mathbf{B}(\boldsymbol{x}, \rho)) \rightarrow \operatorname{Dg}(\mathbf{B}(\boldsymbol{x}, \rho))
$$

as $k \rightarrow \infty$ for each $\rho \in(0, \epsilon) \sim N .{ }^{8}$ Combining with $(7)_{2}$, we then obtain

$$
\left(\mathrm{D} \boldsymbol{g}_{k}-\mathrm{D}_{\mathrm{a}} \boldsymbol{g}_{k} \mathcal{L}^{n}\llcorner\Omega)(\mathbf{B}(\boldsymbol{x}, \rho)) \rightarrow\left(\mathrm{D} \boldsymbol{g}-\boldsymbol{G} \mathcal{L}^{n}\llcorner\Omega)(\mathbf{B}(\boldsymbol{x}, \rho))\right.\right.
$$

as $k \rightarrow \infty$; noting that

$$
\mathrm{D} \boldsymbol{g}_{k}-\mathrm{D}_{\mathrm{a}} \boldsymbol{g}_{k} \mathcal{L}^{n}\left\llcorner\Omega=\left[\boldsymbol{g}_{k}\right] \otimes \boldsymbol{n}_{k} \mathcal{H}^{n-1}\left\llcorner J\left(\boldsymbol{g}_{k}\right),\right.\right.
$$

where $\left[\boldsymbol{g}_{k}\right]$ is the jump of $\boldsymbol{g}_{k}$ on $J\left(\boldsymbol{g}_{k}\right)$ and $\boldsymbol{n}_{k}$ is the normal to $J\left(\boldsymbol{g}_{k}\right)$, we see that (39) reads

$$
\lim _{k \rightarrow \infty} \int_{J\left(\boldsymbol{g}_{k}\right) \cap \mathbf{B}(\boldsymbol{x}, \rho)}\left[\boldsymbol{g}_{k}\right] \otimes \boldsymbol{n}_{k} d \mathcal{H}^{n-1}=\mathrm{D} \boldsymbol{g}(\mathbf{B}(\boldsymbol{x}, \rho))-\int_{\mathbf{B}(\boldsymbol{x}, \rho)} \boldsymbol{G} d \mathcal{L}^{n}
$$

for every $\rho \in(0, \epsilon) \sim N$. This holds for every $\boldsymbol{x} \in \Omega$ where $N=N(\boldsymbol{x})$. Dividing (40) by $\kappa_{n} \rho^{n}$ and using that (16) and

$$
\boldsymbol{G}(\boldsymbol{x})=\lim _{\rho \rightarrow 0} \kappa_{n}^{-1} \rho^{-n} \int_{\mathbf{B}(\boldsymbol{x}, \rho)} \boldsymbol{G} d \mathcal{L}^{n}
$$

hold simultaneously for $\mathcal{L}^{n}$ almost every $\boldsymbol{x} \in \Omega$, we see that for every such an $\boldsymbol{x}$ we have

$$
\lim _{\substack{\rho \rightarrow 0 \\ \rho \in(0, \epsilon) \sim N}} \lim _{k \rightarrow \infty} \int_{J\left(\boldsymbol{g}_{k}\right) \cap \mathbf{B}(\boldsymbol{x}, \rho)}\left[\boldsymbol{g}_{k}\right] \otimes \boldsymbol{n}_{k} d \mathcal{H}^{n-1}=\mathrm{D}_{\mathrm{a}} \boldsymbol{g}-\boldsymbol{G}(\boldsymbol{x}),
$$

i.e., (8) holds.

${ }^{8}$ This is otherwise not a direct consequence of (6). 


\section{Appendix: Elementary proof of a weaker form of the approximation theorem}

We here outline a proof of the following form of the approximation theorem without using Alberti's theorem:

Theorem A.1. If $\Omega$ is an admissible domain and

$$
(\boldsymbol{g}, \boldsymbol{G}) \in B V\left(\Omega, \mathrm{R}^{m}\right) \times L^{1}\left(\Omega, \mathrm{M}^{m \times n}\right),
$$

then there exist two sequences $\boldsymbol{m}_{k}, \boldsymbol{h}_{k} \in S B V\left(\Omega, \mathrm{R}^{m}\right)$ such that

$$
\begin{aligned}
& \boldsymbol{m}_{k} \rightarrow \boldsymbol{g} \text { in } L^{1}\left(\Omega, \mathrm{R}^{m}\right) \text { and } \mathrm{D}_{\mathrm{a}} \boldsymbol{m}_{k}=\mathbf{0} \text { over } \Omega, \\
& \boldsymbol{h}_{k} \rightarrow \mathbf{0} \text { in } L^{1}\left(\Omega, \mathrm{R}^{m}\right) \quad \text { and } \quad \mathrm{D}_{\mathrm{a}} \boldsymbol{h}_{k} \rightarrow \boldsymbol{G} \text { in } L^{1}\left(\Omega, \mathrm{M}^{m \times n}\right) \text {, }
\end{aligned}
$$

and

$$
\sup \left\{\mathrm{M}\left(\mathrm{D} \boldsymbol{m}_{k}\right): k=1, \ldots\right\}<\infty \quad \text { and } \sup \left\{\mathrm{M}\left(\mathrm{D} \boldsymbol{h}_{k}\right): k=1, \ldots\right\}<\infty
$$

consequently, the sequence $\boldsymbol{g}_{k}=\boldsymbol{m}_{k}+\boldsymbol{h}_{k} \in \operatorname{SBV}\left(\Omega, \mathrm{R}^{m}\right)$ satisfies

$$
\boldsymbol{g}_{k} \rightarrow \boldsymbol{g} \text { in } L^{1}\left(\Omega, \mathrm{R}^{m}\right) \quad \text { and } \quad \mathrm{D}_{\mathrm{a}} \boldsymbol{g}_{k} \rightarrow \boldsymbol{G} \text { in } L^{1}\left(\Omega, \mathrm{M}^{m \times n}\right)
$$

and

$$
\sup \left\{\mathrm{M}\left(\mathrm{D} \boldsymbol{g}_{k}\right): k=1, \ldots\right\}<\infty \text { and } \mathrm{D} \boldsymbol{g}_{k} \boldsymbol{\rightarrow}^{*} \mathrm{D} \boldsymbol{g} \quad \text { in } \mathcal{M}\left(\Omega, \mathrm{M}^{m \times n}\right) .
$$

Proof outline. We denote by $\boldsymbol{g}_{0}$ the extension of $\boldsymbol{g}$ to $\mathrm{R}^{n}$ equal to 0 outside $\Omega$. Since $\Omega$ is an admissible domain, we have $g_{0} \in B V\left(\mathrm{R}^{n}, \mathrm{R}^{m}\right)$ by Theorem 3.12. Let $\boldsymbol{f}_{k}$ be a sequence of mollifications of $\boldsymbol{g}_{0}$ on $\mathrm{R}^{n}$ with the mollification parameter tending to 0. Applying Proposition 5.2 in the same way as in the proof of the approximation theorem (Section 1), we find a sequence $\boldsymbol{m}_{k} \in S B V\left(\mathrm{R}^{n}, \mathrm{R}^{m}\right)$ such that $\left|\boldsymbol{f}_{k}-\boldsymbol{m}_{k}\right|_{L^{1}\left(\mathrm{R}^{n}, \mathrm{R}^{m}\right)}<1 / k$. The sequence $\boldsymbol{m}_{k} \mid \Omega$ (again denoted $\boldsymbol{m}_{k}$ ) then satisfies (1) and (3) .

Next, let $\boldsymbol{G}_{0}$ be the extension of $\boldsymbol{G}$ to $\mathrm{R}^{n}$ equal to $\mathbf{0}$ outside $\Omega$, and put

$$
\boldsymbol{h}_{k}(\boldsymbol{x})=\boldsymbol{G}(k, \boldsymbol{p})(\boldsymbol{x}-\boldsymbol{x}(k, \boldsymbol{p}))
$$

for any $\boldsymbol{x} \in \mathrm{R}^{n}$ where $\boldsymbol{p} \in \mathrm{Z}^{n} / k$ is uniquely determined by the requirement $\boldsymbol{x} \in$ $C(k, \boldsymbol{p}), \boldsymbol{x}(k, \boldsymbol{p})$ is the barycenter of $C(k, \boldsymbol{p})$, and

$$
\boldsymbol{G}(k, \boldsymbol{p})=k^{n} \int_{C(k, \boldsymbol{p})} \boldsymbol{G} d \mathcal{L}^{n} .
$$

Then $\boldsymbol{h}_{k}$ is easily seen to satisfy (2) and (3) 2 . 


\section{Acknowledgment}

The author thanks David Owen for his encouragement to complete this work, which was initiated in 2008. The research was supported by RVO 67985840 and by the grant 201/09/0473 of GA ČR. The supports are gratefully acknowledged.

\section{References}

[Alberti 1991] G. Alberti, "A Lusin type theorem for gradients", J. Funct. Anal. 100:1 (1991), 110118.

[Ambrosio et al. 2000] L. Ambrosio, N. Fusco, and D. Pallara, Functions of bounded variation and free discontinuity problems, Oxford University Press, New York, 2000.

[Baía et al. 2011] M. Baía, J. Matias, and P. M. Santos, "A survey on structured deformations”, São Paulo J. Math. Sci. 5:2 (2011), 185-201.

[Choksi and Fonseca 1997] R. Choksi and I. Fonseca, "Bulk and interfacial energy densities for structured deformations of continua”, Arch. Rational Mech. Anal. 138:1 (1997), 37-103.

[Del Piero and Owen 1993] G. Del Piero and D. R. Owen, "Structured deformations of continua", Arch. Rational Mech. Anal. 124:2 (1993), 99-155.

[Del Piero and Owen 1995] G. Del Piero and D. R. Owen, "Integral-gradient formulae for structured deformations", Arch. Rational Mech. Anal. 131:2 (1995), 121-138.

[Del Piero and Owen 2004] G. Del Piero and D. R. Owen (editors), Multiscale modeling in continuum mechanics and structured deformations, CISM Courses and Lectures 447, Springer, Vienna, 2004.

[Evans and Gariepy 1992] L. C. Evans and R. F. Gariepy, Measure theory and fine properties of functions, CRC, Boca Raton, FL, 1992.

[Federer 1969] H. Federer, Geometric measure theory, Die Grundlehren der Mathematischen Wissenschaften 153, Springer, New York, 1969.

[Ziemer 1989] W. P. Ziemer, Weakly differentiable functions: Sobolev spaces and functions of bounded variation, Graduate Texts in Mathematics 120, Springer, New York, 1989.

Received 12 Mar 2014. Revised 11 Jun 2014. Accepted 15 Jul 2014.

MiroslaV ŠILHAVÝ: silhavy@math.cas.cz

Institute of Mathematics, Academy of Sciences of the Czech Republic, Žitná 25, 11567 Prague 1, Czech Republic 